\title{
Pressure Wounds and Skin Failure Secondary Scurvy. Prevention with Early Vitamin Micro Nutrient Supplements
}

\author{
Kaminski MV1* and Mendoza JL2 \\ ${ }^{1}$ American Colleges of Surgeons and Nutrition, USA \\ ${ }^{2}$ Florida State University, USA
}

*Corresponding author: Mitchell V Kaminski, American Colleges of Surgeons and Nutrition 8975 W Golf Rd - Niles IL 60714, USA, Tel: (847) 513-2106; Email: mvkaminski@comcast.net

\section{Research Article \\ Volume 3 Issue 1}

Received Date: November 26, 2018

Published Date: January 07, 2019

DOI: $10.23880 /$ nhij- 16000170

\section{Abstract}

Skin is the largest organ of the body. The dermis is primarily collagen. A collagen deficit results in skin failure with thinning to the point of transparency and disruption. (pressure wounds). The media of blood vessels is also primarily collagen. Here a deficit causes capillary fragility and purpura. A collagen fiber is a triple strand of protein. Plates initially produce three strands of procollagen which twist around each other, first to the left, then right and left again. The result is a fiber more flexible and stronger than a steel wire of the same diameter. Strength is due to cross linking between fibers at the frequent amino acid proline and lysine positions after hydrogenation by vitamin $\mathrm{C}$. It was observed that patients referred for pressure wound care had scurvy at the time of consult. Diagnosing a collagen, i.e. Vitamin C deficit during the admission skin assessment is straight forward by bed side observation for dermal atrophy, purpura or skin tears. If an ample profile of all micro-nutrients with $1 \mathrm{gm}$ of vitamin $\mathrm{C}$ was started along with biofilm control and adequate wound dressings, the existing pressure wounds promptly improved and new wounds were prevented.

Keywords: Vitamin C; Pressure wounds; Prevention; Collagen

\section{Introduction}

The initial work defining Vitamin C deficiency and its requirement for collagen synthesis was published in the late 1930's [1]. As with other vitamin work the guinea pig was used however as with other higher primates, the guinea pig cannot convert glucose into Vitamin C. Therefore, a diet formulated for wound healing research in guinea pigs includes all macro and micro nutrients except Vitamin $\mathrm{C}$.

In humans, acute restriction of Vitamin $\mathrm{C}$ in a young healthy male takes only 160 days to produce classic perifollicular hemorrhages, gingival bleeding and cork screw hair [2]. Therefore, we suggest that in humans, pressure wounds are also associated with chronic under consumption of Vitamin $\mathrm{C}$ which produces pressure wound chronic Scurvy. The hallmarks of which are: dermal atrophy, skin tears and purpura. These three are pathognomonic signs of chronic Pressure wound associated chronic Scurvy and are easily observed on admission skin assessment. Further, it is suggested they form without going through signs of acute Scurvy.

(Figure 1) These signs should be sought for on the mandatory admission skin assessment indicating skin failure, the prologue to pressure ulcers. 


\section{Nursing \& Healthcare International Journal}

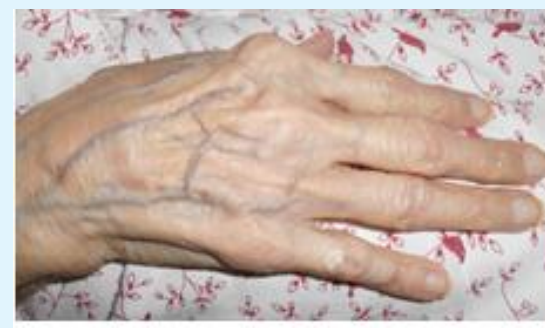

(a)

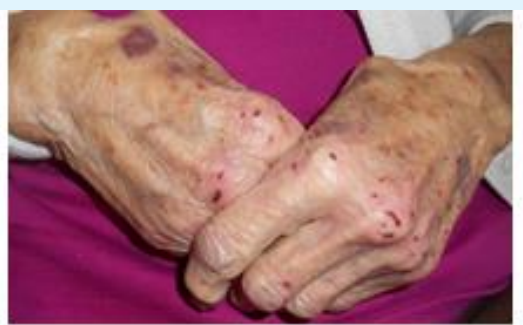

(b)

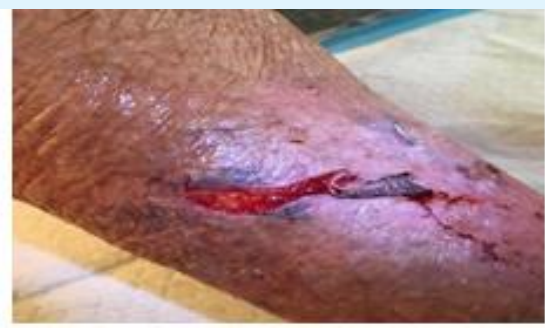

(c)

Figures 1a-c: Chronic scurvy: the typical wasting of the dermis on the dorsum of the hand is illustrated in figure 1a. With the depletion of collagen, the adventitia of the arterioles also atrophies causing capillary fragility leading to purpura (not Coumadin) shown in figure 1b. 1c shows that profound scurvy leaves the skin susceptible to a typical triangular skin tear.

A Vitamin C plasma level will rise or fall rapidly, depending on the size of the dose and the timing of the following blood draw [3]. So, a plasma Vitamin C level can be high or low in a patient with Scurvy. Thus, Scurvy is a clinical diagnosis.

Vitamin C is required for the synthesis of collagen [4]. Collagen is one third of all the formed protein in the body [5]. As with all proteins, collagen undergoes protein turn over. i.e., synthesis vs degradation. If synthesis does not keep up with degradation, over time, a total body collagen deficit will develop [6].

Skin is the largest organ of the body [7]. The dermis of the skin is primarily collagen. The adventitia of the arterioles in the dermis is also collagen. A chronic vitamin $\mathrm{C}$ deficiency causes thinning of the adventitia, capillary fragility and skin transparency where ultimately, the opaque epidermis rests over a diminished dermis exposing the bone and tendons of the hand. Skin tears now can occur with minimal trauma.

It is the collagen in the dermis and media of the arteriole that provides resistance against external pressure. When depleted, external pressure will more easily collapse the remaining dermal capillaries causing blood flow stasis and stasis leads to erythema before clotting [8]. This is a stage I pressure ulcer. When serum leaks beneath the now poorly attached epidermis causing a blister, a stage II wound has formed. Given collagen is concentrated in muscle and tendon, if even minimal pressure continues, the cycle of compression vs diminished resistance/stasis/clotting continues. Stage III and IV wounds develop [9].

The association of a collagen deficit with Scurvy is clear [10]. But, except for this paper, the association of
Scurvy with skin tears is not recognized. The author did a computer search of "skin tears" and found that out of 100 sites, none mentioned Vitamin $\mathrm{C}$ deficiency.

A collagen fiber is a triple strand twine of cross linked protein. It is more flexible and stronger than a steel wire of the same diameter [11]. Collagen is slow to form and degrade. Platelets secret a single protein strand called pro collagen. The amino acids proline and lysine are frequently repeated along its length. Strength is due to twisting and cross linking. This is facilitated by hydroxylation of proline and lysine. Vitamin C hydroxylates proline and lysine [12].

Tissues need to be saturated with Vitamin C to support optimal collagen turnover [13]. Thus, minimal deficiencies over a long periods of time produce deficit accrual. The total body pool of vitamin C is approximately $1500 \mathrm{mg}$. Signs of acute Scurvy occur when it drops to below $300 \mathrm{mg}$ [14].

\section{Materials and Methods}

139 patients referred to the wound care service for work up and management were studied. Attention was paid to the three independent essentials of healing: 1) pressure relief, 2) wound surface care including removal of necrotic debris/biofilm, and 3) use of a moist salubrious dressing, and adequate diet of both macro and micro nutrients. Forearms and hands were also inspected for purpura and skin tears. Observations were recorded on a standardized data collection sheet. A digital camera was used to photograph typical examples of chronic Vitamin C deficiency findings. 


\section{Nursing \& Healthcare International Journal}

Tables 1 \& 2 list the supplements given BID and the laboratory tests done by s certified laboratory at the time

of consult and monthly thereafter.

\begin{tabular}{|c|c|c|c|c|c|c|}
\hline Supplements & Multivitamins & Vitamin C & Zinc Sulfate & Fish Oil & Vitamin B3 & $\begin{array}{c}\text { Glucosamine/ } \\
\text { Chondroitin }\end{array}$ \\
\hline Amount & 1 BIB & $500 \mathrm{mg}$ BID & $220 \mathrm{mg}$ BID & $1 \mathrm{mg}$ BID & $250 \mathrm{mg}$ BID & $400 / 600 \mathrm{mg}$ BID \\
\hline
\end{tabular}

Table 1: List of supplements given all patients cared for on the Wound Care Service BID. The nurse removes the individual tablet from stock bottles, crushes them and mixes the powder with a carrier and serves. Digestive enzymes and a pro-biotic are added as indicated.

Standard Lab Orders (On Consult and each following month)

\section{Vitamin C

\begin{tabular}{|l|l|l|l} 
Vitamin D3 & Zinc & H \\
\hline
\end{tabular} \\ Hemoglobin/
Hematocrit}

\begin{tabular}{|l|l|} 
Transferrin & Ceruloplasmin
\end{tabular}

Table 2: Tests are done regularly to document vitamin C, D3 and Zinc levels. The Hematocrit/Hemoglobin, transferrin and ceruloplasmin were followed to answer an additional question related to the commonly held notion that $440 \mathrm{mg}$ of zinc sulfate/day is toxic and will block absorption of iron and copper causing anemia.

Computer spread sheets were created. The demographic data from each patient along with the physical findings including stage and location of wounds and the patients' laboratory data was entered. A simple mean and standard error were calculated to determine significance between groups and answer the questions asked.

\section{Results}

The figures and tables are typed separately, they are shown in discussion.

\section{Discussion}

Giving a $1 \mathrm{gm} / \mathrm{d}$ Vitamin $\mathrm{C}$ supplement elevated the mean plasma vitamin $\mathrm{C}$ from below normal $(0.34 \mathrm{gm} / \mathrm{dL})$ to just above the lower limits of normal $(0.44 \mathrm{gm} / \mathrm{dL}$ where $0.4 \mathrm{gm} / \mathrm{dL}$ is normal). Figure 2 the dose of $1 \mathrm{gm} /$ day held this plasma level indicating the tissues are saturated. Further indicating that $1 \mathrm{gm}$ is adequate, the author has noted that a skin tear rapidly heals at this dose. Within five to seven days, the epidermis that has been repositioned over its dermis will firmly knit to it. In the author's experience, this does not occur if at least $1 \mathrm{gm} / \mathrm{d}$ vitamin $\mathrm{C}$ is given to the patient.

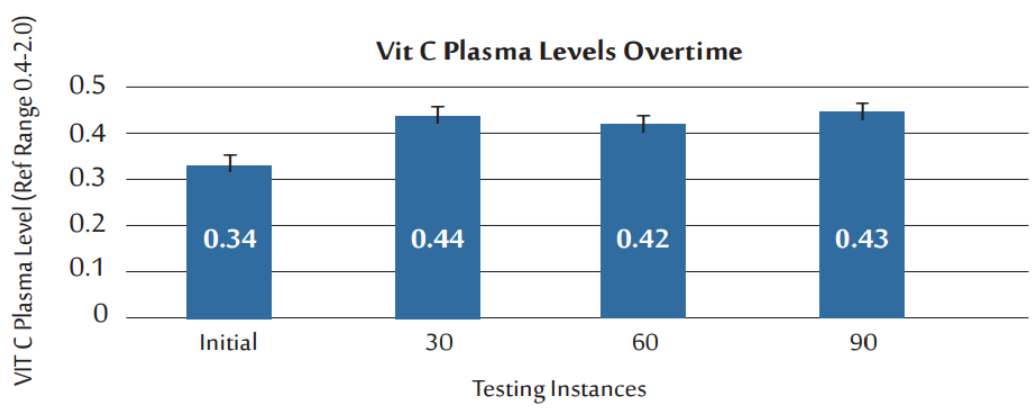

Figure 2: The mean plasma Vitamin C for patients was calculated on initial consult, 30 days, 60 days, then 90 days after initial consult to ascertain the serial changes of Vitamin $C$ during supplementation. The dotted line indicates the beginning of the healthy range of Vitamin C levels $(.4 \mathrm{mg} / \mathrm{dL}) .19$ patients were not included in the count because their initial labs did not include Vitamin $\mathrm{C}$. Initial $\mathrm{N}=117 ; 30$ days $\mathrm{N}=40 ; 60$ days $\mathrm{N}=28 ; 90$ days $\mathrm{N}=14$.

It is best to use only a single $1 / 4$ in adhesive strip at the apex of the triangular tear to fix the epidermis in position. Pus will form under the adhesive strips if more are used. Do not use any other adhesive products on 
"Scurvy Skin". They will potentially remove the epidermis adherent to the adhesive side when it is stripped. Use a non-adherent dressing and a gauze wrap. Placing a small amount of maltodextrin on the remaining exposed dermis seems to speed recovery [15] (Figure 3).

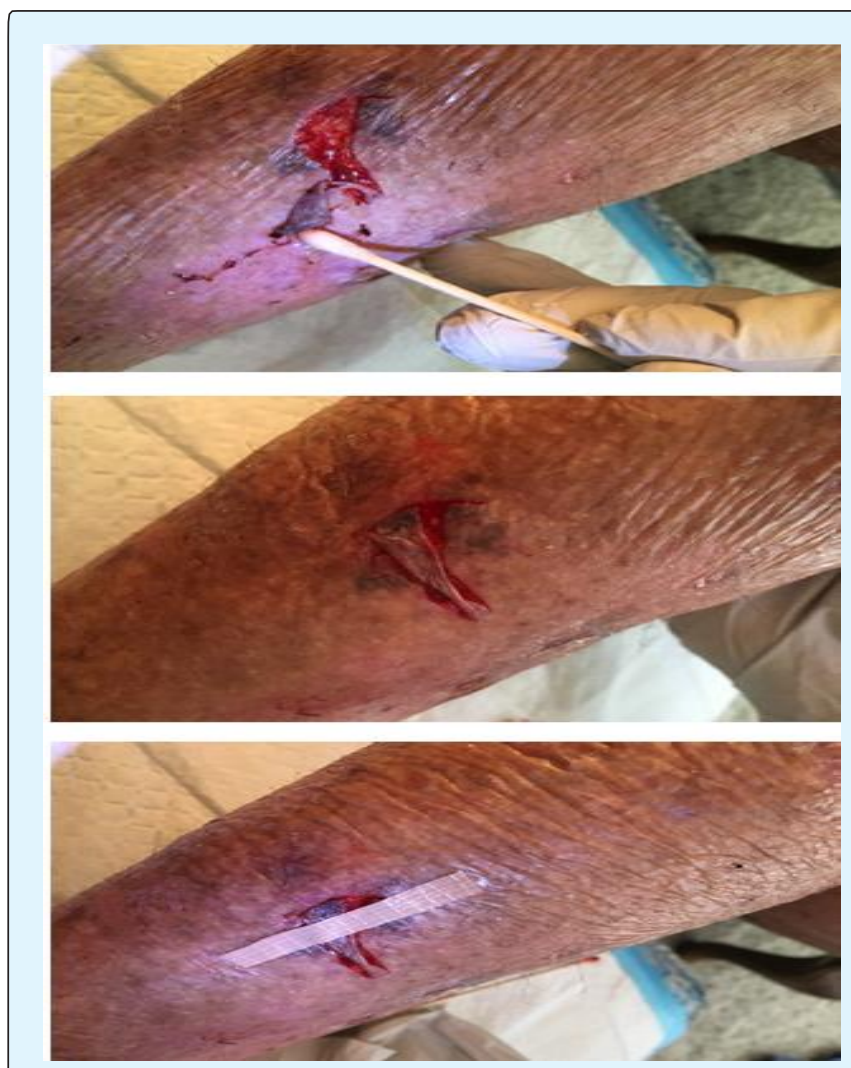

Figure 3: The flap should be repositioned as a split thickness skin graft held in place by a steri-strip. Not more than one steri-strip should be used as a purulent discharge may accumulate under more. This is a sign of scurvy and not a condition in itself. Oral vitamin $C$ is mandatory. Further dressings with adhesive surfaces may cause further tearing of the epidermis.

Unlike the diagnosis of anemia, where the signs and symptoms are confirmed by hematocrit/hemoglobin, a laboratory value, Scurvy cannot be confirmed by a plasma vitamin $C$ level. What would be required would be a total body collagen assessment. The correlation of the signs of chronic Scurvy with vitamin C plasma levels below normal are helpful and intuitively make sense and may be of significant value.

For example, they serve to question the adequacy of dietary micronutrients in general. The conclusion should be that all patients with pressure wounds have Scurvy first.

Further evidence contributing to the impression that Scurvy precedes the formation of pressure wounds is that whether the pressure wound was stage II or IV, no group at the time of consult had a plasma Vitamin $C$ within the normal range, and the plasma vitamin $C$ was predictable (Figure 4).

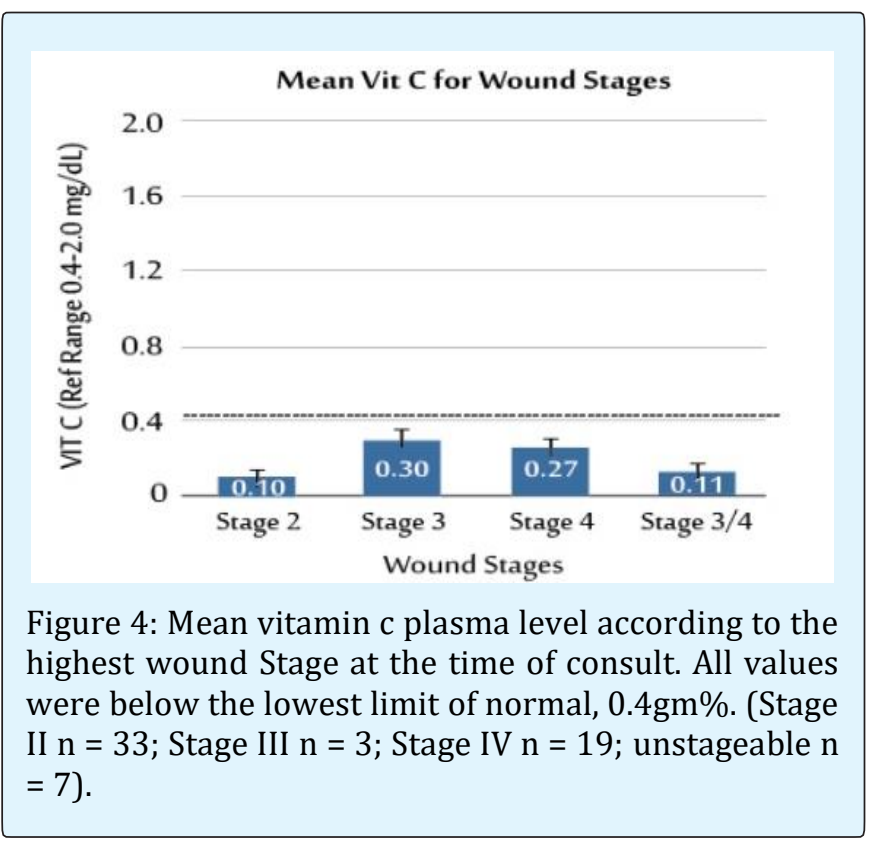

Recovery of accrued collagen deficits take time. In the author's experience, 6 to 9 months is required to begin to see the dermis become noticeably thicker on the dorsum of the hands and temporal area.

Plasma vitamin $\mathrm{C}$ over time shows a nonlinear association of the plasma Vitamin $\mathrm{C}$ level with the formation or progression of a wound. Figure 2 although plasma Vitamin $\mathrm{C}$ levels rise slightly with supplementation as illustrated, it shows that recovery from accrued collagen deficits is lengthy. All patients with pressure wounds have Chronic Scurvy by physical exam at the time of consult which is tightly correlated only with a below normal Vitamin $\mathrm{C}$ value.

This raises the question of when to start cutaneous support supplements; when a wound forms or when chronic vitamin $\mathrm{C}$ deficiencies are first documented?

Can giving vitamin $\mathrm{C}$ at $1 \mathrm{gm} /$ day as part of a comprehensive care plan for patients with wounds have a 
positive effect on indolent wounds? One gran is given because trusses need to be saturated. The author reports that, in combination with other critical micronutrients and a comprehensive wound care protocol, wounds quickly change form indolent to healing. For example, a skin tear will reattach the epidermis to dermis using a single adhesive strip to hold the epidermal flap in place in 5 to 7 days. Stage III and IV wounds quickly evolve to 100\% granulation with no odor, drainage or biofilm. However, the total protocol involves other critical elements such as pressure relief and mechanical removal of necrotic debris and biofilm.
Grouping the patients by the cutaneous signs of chronic Scurvy, purpura, thin dermis $3+/ 4+$ vs skin tear $3+/ 4+$ vs pressure wounds stage III/IV shows a mean plasma $\mathrm{C}$ level to be the same for purpura, skin tears and pressure wounds. Figure 5 it appears that once scurvy is established, how it manifests' a wound depends on external physical circumstances. The signs have to do with a total body collagen deficit, not a below normal plasma vitamin C.

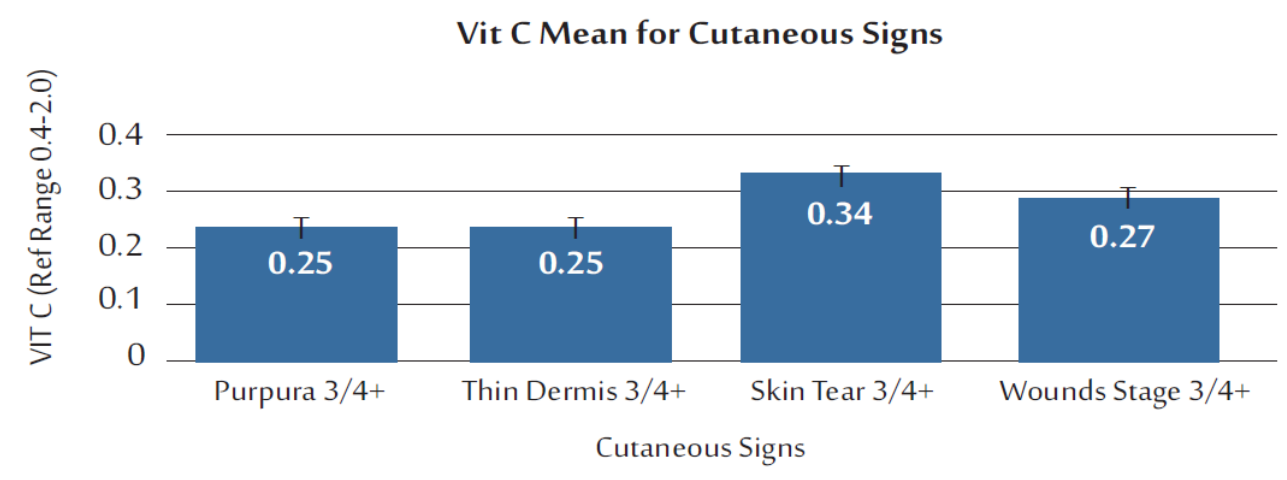

Figure 5: The mean Vit $\mathrm{C}$ was assessed for each of the cutaneous signs of possible Vit $\mathrm{C}$ deficiency. The dotted line indicates the beginning of the healthy range of Vit $\mathrm{C}$ levels $(0.4 \mathrm{mg} / \mathrm{dL})$. Purpura $3 / 4+\mathrm{N}=8$; Thin Dermis $3 / 4+\mathrm{N}=48$; Skin Tear N=11; Wound Stage 3/4 + N=19

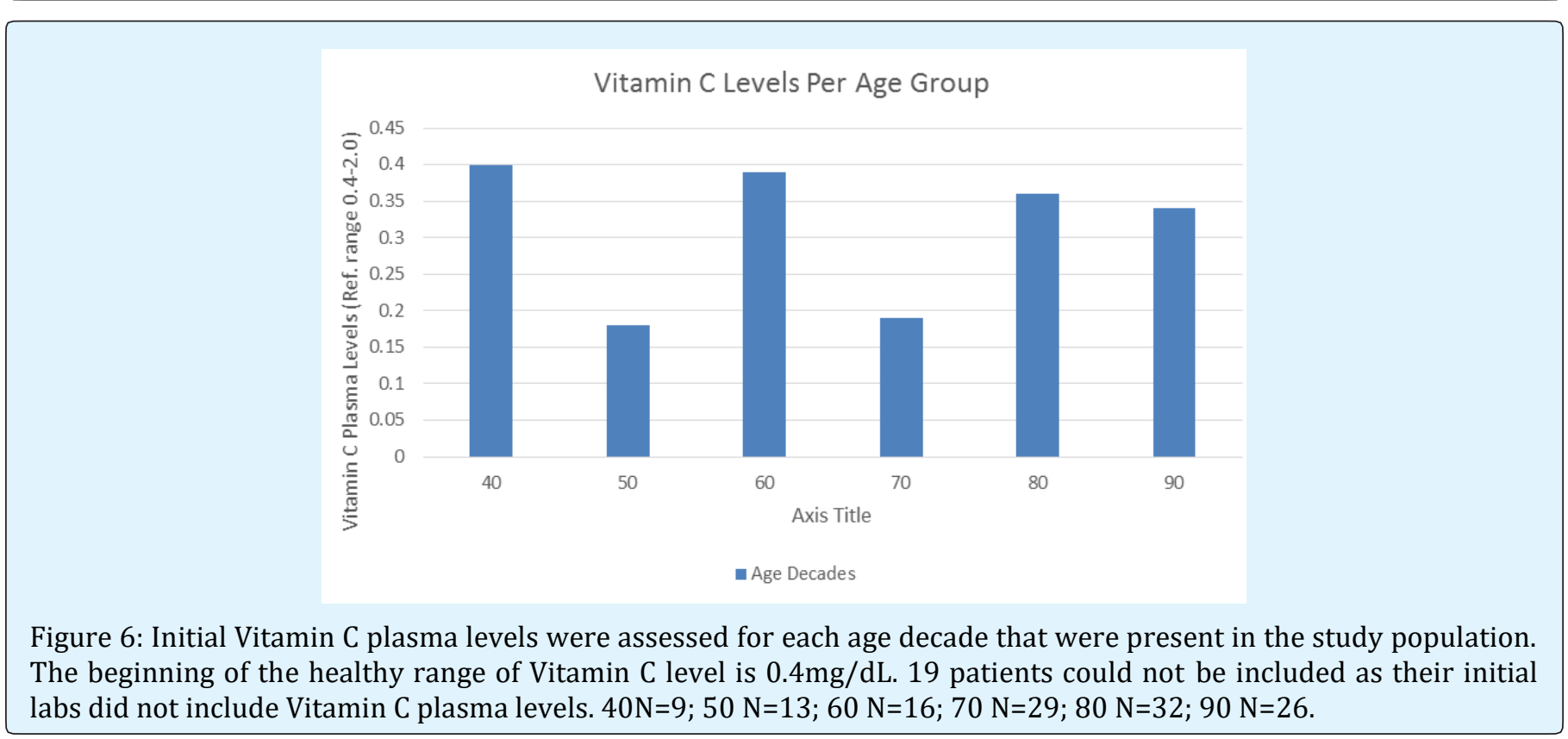


Thus, if the patient was being treated for a skin tear which has now healed, vitamin C supplements must continue. Recall, collagen is slow to synthesize and the deficit is great. Do not stop the supplement if the plasma level happens to be in normal range on any single laboratory draw.

As one ages, malabsorption of macro and micronutrients are known to be an issue [16]. Perhaps it is true for vitamin C. To test this, the patients were grouped according to decade of life. Figure 6 except for the $4^{\text {th }}$ decade, all other groups were below normal and a decreasing mean vitamin $\mathrm{C}$ pattern was observed as age increased. This is not proof that giving enzyme supplements would not help, which is a trial that needs to be done.

The literature does not currently recognize chronic scurvy. Figure 6 demonstrates plasma Vitamin C levels did not correlate with skin tears located at various sites or advanced pressure wounds stages III/IV. This is a recurrent finding. i.e., low plasma levels do not necessarily correlate with wounds or healing. The patient is predisposed to forming a wound by a progressive vitamin $\mathrm{C} /$ collagen depletion and structural coetaneous failure. It is the location and intensity of the external pressure against the integrity of the remaining collagen in the dermis and the arterioles that results in a wound.

These data are consistent in that low and below normal plasma Vitamin C levels are characteristic of nursing home patients with pressure wounds. Obtaining and tracking plasma $\mathrm{C}$ levels are also helpful to assure that $1 \mathrm{gm}$ of Vitamin $\mathrm{C}$ per day is not an overdose. The theoretical goal is to keep increasing the dose until a predictable retention is identified. Vitamin $\mathrm{C}$ is water soluble, non-toxic and inexpensive but plays a critical role in wound healing. A wound will not heal, in fact regress in scorbutic patients [2].

Currently, the literature dose not identify the cutaneous triad of findings; 1) thin dermis 2) purpura and 3) skin tears as characteristic signs of chronic Scurvy.

Rather these signs are variously called Senile Purpura and occasionally actinic purpura (UV radiation damage) and even simply ecchymosis. However, by observing the surface skin texture of the hands vs the face, bleeding into tissues, and plasma vitamin $\mathrm{C}$ one can distinguish chronic Scurvy from actinic keratosis from a bruise (Figure 7).

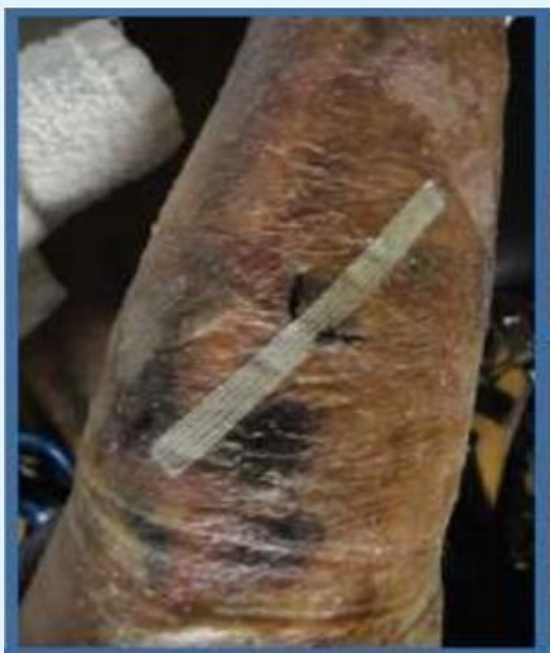

A. Purpura

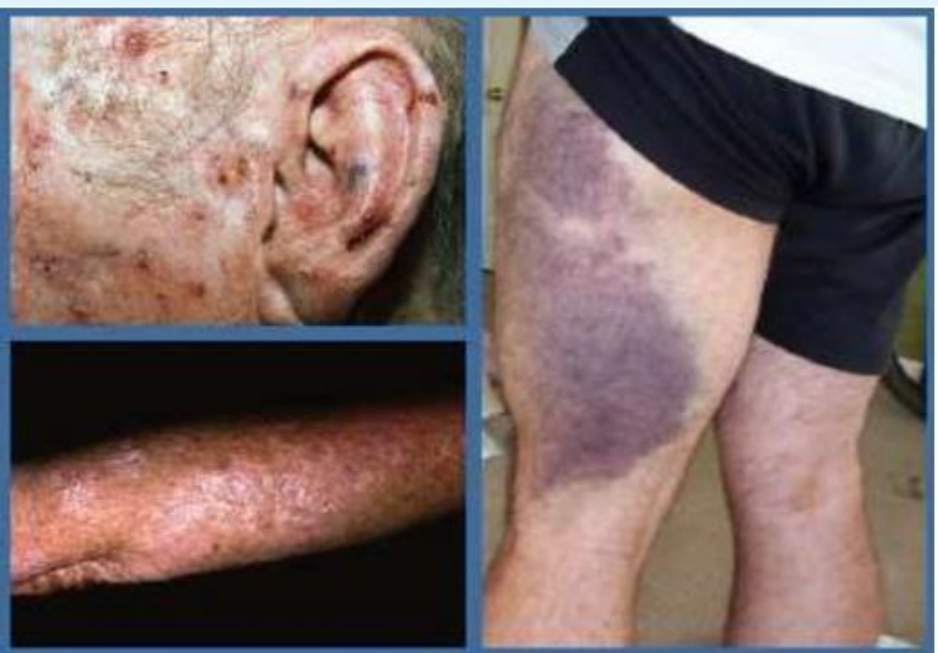

B. Face/forearm
C. Bruise

Figure 7: A- Purpura with skin tear. B- Actinic keratosis of the face and forearm due to excessive sun exposure. CCapillary trauma with dermal bleeding.

In acute scurvy, a fair amount of normal dermis is present. The vessels around the hair follicle are thin and forming chiastic perifollicular hemorrhages and cork screw or goose neck hairs. A plasma Vitamin C can be normal to below normal depending on when the blood was drawn in relation to the dose and time from the last ingestion of food containing Vitamin C. However, the face is spared. 


\section{Nursing \& Healthcare International Journal}

Senile purpura is chronic Scurvy. The surface of the skin is thin and smooth. Bleeding is between the epidermis and dermis. Once again plasma Vitamin C reflects the last ingestion of Vitamin $\mathrm{C}$ and the timing of the blood draw. Careful inspection of the skin of the face in the temporal area might reveal thinning of the dermis with a transparent epidermis.

Actinic keratosis is sun damage from chronic exposure to UV radiation. The skin surface is rough and small blood vessels may be viable as flairs but there is no evidence of sub epidermal bleeding. Plasma Vitamin C levels are usually normal as Vitamin C intake is not the problem.

There are those who mistake skin changes on the face are due to sun exposure. The key observation is that along with the hands, the sun exposed area of the face is involved. The roughened areas can be precancerous (Figure 7).

An ecchymosis is a bleed into the soft subcutaneous tissues below the dermis. The overlying skin is smooth, and Vitamin C is normal. This is a process located at the site of trauma, so the face is not involved unless the trauma was to the head.

In the authors experience, the findings associated with chronic Scurvy are common, especially in the geriatric group.

How can a vitamin $\mathrm{C}$ deficient diet occur prior to admission to a nursing home?

Fresh fruits and vegetables are the source of Vitamin C and other micronutrients. Their purchase and consumption decrease with age, typically with the loss of a spouse. Now, eating is no longer a social event and the patient will say: "they spoil too quickly and are too expensive". Also, canning will destroy vitamins and finally, much to the discredit of the medical profession, patients are advised by their doctors that they do not need to take vitamin supplements as long as they are eating a "normal diet". And, supplements are "just creating expensive urine". This was refuted decades ago in an article in the Journal of the American Medical Association [17].

In 1940 a medical student conducted a Vitamin C deprivation study diet. The author suggests you call to mind your experience with patients observed to have signs of pressure wound associated chronic Scurvy. During this experiment the plasma content of ascorbic acid fell to a low level within 10 days and disappeared in
30 days; after 132 days on the Vitamin C deficient diet the first abnormal objective clinical signs were observed. The Vitamin C content of the white cells/platelet layer of centrifuged blood could be correlated much more directly with development of the disease. This value fell to 0 shortly before developing scurvy. Another early sign was the finding of interruption of the lamina dura in $\mathrm{x}$-ray films of the teeth. The earliest clinical manifestations of scurvy were hyperkeratotic papules which were noted after 132 days on the Vitamin $\mathrm{C}$ deficient diet; the perifollicular hemorrhages of scurvy required another month (161 days) for their appearance. There was a noticeable fragmentation of hair associated with these signs was a pronounced dryness of the skin particularly over the extensor surfaces. Subsequent weakness was noted by the end of the third month and increased progressively. The subject suffered a steady and progressive weight loss of 12 kilos. Blood pressure also fell. Anemia did not occur. Wound healing was studied by means of biopsies. The first sign of deficient wound healing was three months after the start of the experiment. At this time normal wound healing occurred although the plasma ascorbic acid level had been 0 for 44 days. The second test was made at the end of 182 days. Definite failure of healing was noted at this time. beneath the surface of the skin which appeared healed but was an unorganized blood clot. Microscopically the tissue showed a lack of intracellular and capillary function. A wound form an appendectomy healed for 11 years suffered a spontaneous disruption [18].

\section{Conclusion}

These data suggest Scurvy precedes formation of a pressure wound. Therefore, the admission skin assessment should include evaluation of the dorsum of the hand and forearms for signs of chronic scurvy. If found, the patient should be started on a coetaneous support supplement profile which contains $1 \mathrm{gm}$ of Vitamin C. A deficiency in one micronutrient means there are deficiencies across the board.

Future work: Prior to this report, signs of chronic Scurvy have gone unrecognized and may indicate the need for early micronutrient supplement intervention. This paper connects physical signs of a collagen deficit of the dorsum of the hands/forearms with below normal plasma vitamin C plasma levels with pressure wounds. Since plasma vitamin C deficits were documented at the time of wound consult, Scurvy existed prior to the wound and may have played a seminal role. 


\section{Nursing \& Healthcare International Journal}

A multicenter study is suggested. The hypothesis to be tested is: Early detection of a collagen deficit followed by micronutrient supplement intervention (including adequate vitamin $\mathrm{C}$ ) will reduce the incidence of pressure ulcers.

The Director of Nursing at one of the facilities the author covered for wound care was enthusiastic about the positive results she observed using the Three Pillar Protocol [19]. She suggested that when the admitting nurse observed signs of Scurvy, the primary care physician be contacted and a multivitamin be ordered BID plus $1 \mathrm{gm}$ of vitamin $C$ per day [20]. The incidence of pressure wounds was tallied weekly on Mondays. After nine months the weekly incidence of pressure wounds dropped from four to eight down to zero to two.

\section{References}

1. Mettier SR, Minot GR, Townsend WC (1930) Scurvy in Adults. Journal of the American Medical Association 95(15): 1089-1093.

2. Hirschmann J, Raugi GJ (1999) Adult scurvy. J Am Acad Dermatol 41(6): 895-910.

3. Levine M, Conry Cantilena C, Wang Y, Welch RW, Washko PW, et al. (1996) Vitamin C pharmacokinetics in healthy volunteers: evidence for a recommended dietary allowance. Proc Natl Acad Sci U S A 93(8): 3704-3709.

4. Gershoff SN (1993) Vitamin C (Ascorbic Acid): New Roles, New Requirements? Nutrition Reviews banner. 51(11): 313-326.

5. McIntosh J, Webberly H (2015) What is Collagen? What does Collagen Do? Medical News Today.

6. Chojkier M, Spanheimer R, Peterkofsky B (1983) Specifically decreased collagen biosynthesis in scurvy dissociated from an effect on proline hydroxylation and correlated with body weight loss. In vitro studies in guinea pig calvarial bones. J Clin Invest 72(3): 826835.

7. Slominski A, Wortsman J, Paus R, Elias PM, Tobin DJ, et al. (2008) Skin as an endocrine organ: implications for its function. Drug Discovery Today: Disease Mechanisms 5(2): 137-144.
8. Aronson D (2003) Cross-linking of glycated collagen in the pathogenesis of arterial and myocardial stiffening of aging and diabetes. J Hypertens 21(1): 312.

9. NPUAP Pressure Injury Stages (2016) The National Pressure Ulcer Advisory Panel - NPUAP. The National Pressure Ulcer Advisory Panel NPUAP.

10. Kip D, Melvin M, Kimmel D, Akhter M, Robinson R, et al. (1996) Scurvy results in decreased collagen synthesis and bone density in the guinea pig animal model. Bone 18(3): 281-288.

11. Lodish H (2000) Collagen: The Fibrous Proteins of the Matrix. In: Zispursky B, et al. (eds), Mollecular Cell Biology. $4^{\text {th }}$ (Edn.), Freeman WH \& Company.

12. Libby P, Aikawa M (2002) Vitamin C, Collagen, and Cracks in the Plaque. Circulation 105(12): 1396-1398.

13. Gerster H (1987) Human vitamin $C$ requirements. $Z$ Ernahrungswiss 26(2): 125-137.

14. Fain $O$ (2005) Musculoskeletal manifestations of scurvy. Joint Bone Spine 72(2): 124-128.

15. Multidex $\AA$ Gel or Powder (2016) Multidex $\AA$ Gel or Powder | Wound Care Filler Dressing Products.

16. Russell R (2001) Factors in aging that effect the bioavailability of nutrients. The American Society for Nutritional Sciences 131(4): 1359S-1361S.

17. Gaziano JM, Sesso HD, Christen WG, Bubes V, Smith JP, et al. (2012) Multivitamins in the prevention of cancer in men: the Physicians' Health Study II randomized controlled trial. JAMA 308(18): 18711880.

18. Crandon J, Lund C, Dill D (1940) Experimental Human Scurvy. Journal of the American Medical Association 115(19): 1637.

19. Kaminski MV, Mendoza JL (2018) Pressure Wound Healing The Three Pillar Protocol. Nursing \& Healthcare International Journal 2(4): 000152.

20. Kaminski MV, Drinane JJ (2014) Learning the Oral and Cutaneous Signs of Micronutrient Deficiencies. J Wound Ostomy Continence Nurs 41(2): 127-135.

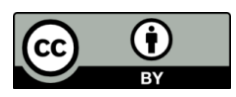

Kaminski MV and Mendoza JL. Pressure Wounds and Skin Failure Secondary Scurvy. Prevention with Early Vitamin Micro Nutrient Supplements. Nurs Health Care Int J 2019, 3(1): 000170. 